\title{
Two novel biallelic mutations in PSMC3IP in a Chinese patient affected by primary ovarian insufficiency: case report and review of the literature
}

Libin Mei ( $\square$ meilibinxm@163.com )

Xiamen University

\section{Lingling Huang}

Xiamen University Medical College

\section{Yanru Huang}

Xiamen University Medical College

Xiaoling Wu

Xiamen University Medical College

Huang He

Xiamen University Medical College

\section{XueMei He}

Xiamen University Medical College

\section{Zhiying Su}

Xiamen University Medical College

\section{Ping Li}

Xiamen University Medical College

\section{Research}

Keywords: premature ovarian insufficiency, PSMC3IP, biallelic mutations, whole-exome sequencing

Posted Date: December 16th, 2020

DOI: https://doi.org/10.21203/rs.3.rs-33852/v2

License: (c) (i) This work is licensed under a Creative Commons Attribution 4.0 International License. Read Full License 


\section{Abstract}

Background: Premature ovarian insufficiency (POI) is a heterogeneous condition occurring when a woman experiences a loss of ovarian activity before the age of 40 . It is one of the most common reproductive endocrine diseases in women of childbearing age. Here, we investigated the clinical manifestations and genetic features of a Chinese patient affected by POI.

Methods: We applied next-generation whole-exome capture sequencing with Sanger direct sequencing to the proband and her clinically unaffected family members.

Results: Two novel compound heterozygous mutations were identified in the PSMC3IP gene. The first is a splicing mutation (c.597+1G $>T$ ) that was inherited from her father, while the second mutation (c.268G $>C$ p.D90H) was also discovered in her mother and younger sister. The two mutations were co-segregated with the disease phenotype in the family.

Conclusions: To our knowledge, this is the first report of PSMC3IP mutations causing POI in the Chinese population. Our findings further support the key role of the PSMC3IP gene in the etiology of POI. However, additional studies are required to explore the underlying molecular mechanisms involved.

\section{Background}

Premature ovarian insufficiency (POI) refers to the presence of ovarian atrophic permanent amenorrhea in women under the age of 40 , characterized by hypergonadotropic hypogonadism and presenting with either primary or secondary amenorrhea [1]. In women of reproductive age, $\mathrm{POI}$ is one of the most commonly diagnosed endocrine diseases. The prevalence of $\mathrm{POI}$ is approximately $1 \%$ [2]. Apart from menstrual disturbance, the main symptoms of $\mathrm{POI}$ are the decrease of estradiol levels and the increase of plasma follicle-stimulating hormone levels (FSH) $(>25$ $\mathrm{mlU} / \mathrm{ml}$ on two occasions, $>4$ weeks apart) $[3,4]$.

The etiology of POI is highly heterogeneous and complex, such as that for genetic, autoimmune, infectious, and iatrogenic factors, among which genetic causes explain presentation in approximately $20-25 \%$ of patients [5]. Over the past few years, novel methods using next-generation sequencing (NGS), particularly whole-exome sequencing (WES), have led to the identification of numerous candidate genes. These genes are mainly involved in meiosis, DNA damage repair, and homologous recombination, including X-linked genes (e.g. FMR1, BMP15, and PGRMC1) and autosomal genes (e.g. FSHR, NOBOX, FIGLA,GDF9, FOXL2, and STAG3) [5-7]. In 2011, WES revealed PSMC3IP as a novel candidate gene associated with autosomal recessive $\mathrm{POI}$ [8]. PSMC3IP is important for homologous pairing and homologous recombination in meiosis, which is indicated by its yeast ortholog HOP2. Female PSMC31P/HOP2deficient mice displayed a significantly reduction in ovarian volume and a lack of follicles[8,11]. To date, rare variants of the PSMC3IP gene were reported in POI[8-10].

Here, we present the case study of an adopted Chinese woman suffering from POI. We performed WES on the patient's DNA to identify potential causative genes or mutations of PSMC3IP associated with POI. Identified sequences were subjected to extensive bioinformatical analysis and screening against several databases to predict the potential effect on protein function. Mutations were confirmed with Sanger sequencing and screened against negative control DNA.

\section{Materials And Methods}




\subsection{Clinical case report}

The proband, a 29-year-old female from Fujian(Fig 1 ), China, who had primary amenorrhea, had been married for 5 years without conceiving and had been diagnosed with POI. She had a normal target height $(160 \mathrm{~cm})$, and normal weight (55kg). Physical examination showed no dysmorphic features or breast development and normal intellectual development. ynaecological examination, it was clear that the patient had a sparse amount of pubic and armpit hair. A transvaginal ultrasound examination revealed that the bilateral ovaries were abnormally small,the left ovary was $1.23 \times 1.00 \mathrm{~cm}$, and the right ovary was $1.55 \times 0.74 \mathrm{~cm}$, but no obvious antral follicles were observed(Fig 2). Her basic hormone levels were as follows: FSH 62.52-78.60 IU/L, Luteinizing hormone (LH) 20.38-25.43 IU/L, estradiol (E2) 13.0-42.5 pmol/L, and anti-Müllerian hormone (AMH) $0.09 \mathrm{ng} / \mathrm{ml}$. She had a normal 46,XX karyotype and $F M R 1$ repeat lengths and a negative testing of adrenal cortical antibody. The biological parents of the proband were healthy and non-consanguineous. The proband's biological mother and sister had normal menstrual histories, and the family did not report any history of systemic diseases or solid tumors. This study fully complied with the tenets of the Declaration of Helsinki and has been approved by the Ethics Board of the Women's and children's Hospital affiliated to Xiamen University, China. Informed consent was obtained from all participants before testing.

One hundred unrelated ethnically matched healthy female individuals (aged between 22 and 40, average age of 28) were recruited as controls, They were known to be menstruating regularly, had normal FSH levels and normal pelvic ultrasound imaging. .

\subsection{Targeted exon capturing and next-generation sequencing}

Total genomic DNA was extracted from peripheral blood leukocytes using the Blood Genomic DNA Mini Kit (Qiagen, Valencia, CA, USA). The concentration of DNA samples was analyzed using a NanoDrop 2000 spectrophotometer (Thermo Fischer Scientific, Waltham, MA, USA).The exomes were captured with SureSelect Human All Exon V6 (Agilent Technologies, Santa Clara, CA, USA) and sequenced on an average depth of $150 \mathrm{x}$ with the use of the Illumina HiSeq platform (Illumina, San Diego, CA, USA) according to the manufacturer's protocol.

\subsection{Bioinformatic analysis}

After Illumina HiSeq sequencing, raw NGS data were imported into FastQC software for assessing the quality and the high-quality reads were aligned to the human reference genome (GRCh37/hg19) using Burrows-Wheeler Aligner (BWA) software. Then, variant calling and annotation were performed using GATK software. Several databases, such as the Single Nucleotide Polymorphism Database (dbSNP)138, the 1000 Genome Project, the Exome Aggregation Consortium (ExAc), ClinVar, and the Genome Aggregation Database (gnomAD) were employed to select all variants with frequencies higher than 5\%. In addition, online tools such as Human Splicing Finder囚PolyPhen-2】and SIFT were applied to predict the potential effect on protein function.

\subsection{Confirmation by Sanger sequencing}

The mutations of the PSMC3IP gene were further confirmed by Sanger sequencing. The PCR products were sequenced on an ABI 3730xI DNA Analyzer (Applied Biosystems, Thermo Fisher Scientific). Sequencing results were analyzed using the DNASTAR Lasergene software (DNASTAR, Madison, WI, USA). Subsequently囚the novel identified mutations were verified and screened in 100 unrelated healthy women (aged between 22 and 40, average age of 28) with DNA sequencing.

\section{Results}




\subsection{Mutation identification by next-generation sequencing and Sanger sequencing}

Overall, the coverage of the target region was $99.3 \%$ with an average sequencing depth of more than 130x and with a variant accuracy of more than $99.97 \%$. After filtering out all the existing mutations with a minor allele frequency (MAF) greater than 0.05 as determined with dbSNP138, 1000 Genomes, ExAc, ClinVar, and gnomAD, a total of 18 variants were remaining(Supplementary Table 1).

In combination with the clinical phenotype and database analyses, two compound heterozygous mutations of PSMC3IP, c.597+1G >T and c.268G>C, were considered as pathogenic compound heterozygous mutations.

Furthermore, Sanger sequencing on the family members' DNA confirmed that the c.597+1G>T mutation was inherited from the father $\$ while the missense mutations $(c .268 \mathrm{G}>C$ ) was also observed in her mother and younger sister, showing complete co-segregation of the mutations with the disease phenotype(Fig 3).

\subsection{Prediction of the pathogenic significance of the mutations}

According to the classification standards of American College of Medical Genetics and Genomics (ACMG), c. $597+1 \mathrm{~g}>\mathrm{T}$ and $\mathrm{c} .268 \mathrm{~g}>\mathrm{C}$ were classified as suspected pathogenic mutations. The splicing mutation c. $4106+2 \mathrm{~T}>\mathrm{C}$ was predicted to alter the splice donor site, most likely influenced by splicing, according to Human Splicing Finder (http://www.umd.be/HSF3/HSF.shtml/). The c.268G $>$ C mutation is a missense mutation and results in a substitution of aspartate with histidine at amino acid position 90 (p.D90H). According to Clustal W/Ensembl software (UCD Conway Institute, Dublin, Ireland), species conservation analysis confirmed that the ninetieth aspartic acid residues were highly conserved among different species(Fig $\mathbf{4}$ b). The mutation was described as "possible damage" by using online disease prediction software PolyPhen-2(Fig 4 a), and "Affect Protein Function" by SIFT. Neither of the two mutations has been reported in the Human Gene Mutation Database (HGMD), dbSNP138, the ExAC database, the 1000 Genomes database, or in any other single-nucleotide polymorphism database. In addition, no relevant literature has been reported. Moreover, neither of the heterozygous mutations were found in 100 unrelated control individuals from the same ethnic origin (data not shown). Taken together, these results powerfully support that PSMC3IP mutations are disease causing mutations in the family.

\section{Discussion}

We evaluated an adopted 29-year-old Chinese woman with POI and identified two biallelic mutations, c.597+1G>T and c. $268 \mathrm{G}>\mathrm{C}$, in the PSMC3IP gene. The two mutations carried by the patient were inherited respectively from her biological mother and father. PSMC3IP has previously been linked to hereditary breast and ovarian cancer, as well as causing autosomal recessive POI $[8,12,13]$. PSMC3IP defects can disrupt estrogen-driven transcription activation of PSMC3IP. Impaired estrogenic signaling can result in ovarian dysgenesis by interfering the follicular pool and against counteracting follicular atresia $[8,14]$.

The PSMC3IP gene is located at 17q21.2, consist of 217 amino acids in its monomer, encoding a nuclear, tissuespecific protein with multiple functions, including a role in meiotic recombination and acting as a coactivator of ligand-dependent transcription mediated by nuclear hormone receptors, which is conserved in evolution $[15,16]$. Previous studies proved that in the PSMC3IP knockout model of mice, the ovarian volume is reduced and the germ cells are missing[17,18]. PSMC3IP is a DNA-binding protein dime, characterized by the presence of three domains including a leucine zipper domain, a DNA-binding domain, and a RAD51/DMC1 interaction domain [9]. The c.268G>C mutation occurs within the highly conserved leucine zipper domains(Fig $4 \mathrm{c}$ ). In vitro experiments showed that a defect of the leucine zipper eliminated the dimerization of PSMC3IP [15]. The c.268G $>C$ mutation was detected in the proband's mother and sister with normal ovarian function. The splicing mutation c.597+1G>T is predicted to alter the 
splice donor site so that most likely to interfere with splicing. However, the exact effects of splice site mutations on mRNA cleavage are not clear and need to be investigated further.

To date, only four POI families with PSMC3IP mutations have been described worldwide, including one reported in

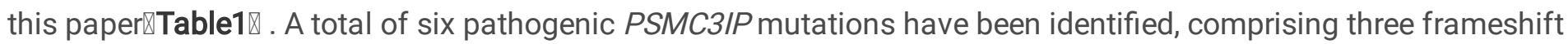
mutations, one nonsense mutation, one missense mutation, and a splicing mutation. In 2011, Zangen et al. [8] first identified a homozygous 3 bp in-frame deletion in the exon 8 of PSMC3IPgene in a large consanguineous Arab Palestinian pedigree with XX-female gonadal dysgenesis, leading to the deletion of glu201. Also, in a consanguineous Yemeni family of a brother with azoospermia and four sisters with ovarian dysgenesis, Abdulmoein et al. [9] identified a homozygous stop mutation (c.489C>G, p.Tyr163Ter) in PSMC3IP, suggesting an important role of PSMC3IP in the development of male and female germ cells. In our study, the proband's father and carried the heterozygous splice site mutation c.597+1G>T , but he didn't show spermatogenesis dysfunction. Recently, two compound heterozygous mutations of PSMC3IP (c.430_431insGA, p.L144*; c.496_497deICT, p.R166Afs) were found in a 28-year-old French female who presented with POI [10]. However,Norling A et al. reported that PSMC3IP gene mutations are not common causes of POI in this Swedish cohort. The pathogenesis of POI caused by PSMC3IP needs to be further verified in the future. Since the limited number of cases of PSMC3IP mutations associated with POI, we are not able to make a clear correlation between this genotype and phenotype.

Table 1

\begin{tabular}{|c|c|c|c|c|c|c|c|c|}
\hline Family & $\begin{array}{l}\text { Age at } \\
\text { diagnosis }\end{array}$ & $\begin{array}{l}\text { Ethnic } \\
\text { origin }\end{array}$ & CS & karyotype & $\begin{array}{l}\text { Nucleotide } \\
\text { change }\end{array}$ & $\begin{array}{l}\text { Amino acid } \\
\text { change }\end{array}$ & Status & Reference \\
\hline 1 & 21 & Palestinian & Yes & $46, X X$ & c.[600_602del] & p.Glu201del & ho & $\begin{array}{l}\text { Zangen } \\
\text { et al., } \\
(2011)\end{array}$ \\
\hline 2 & 28 & French & No & $46, X X$ & $\begin{array}{l}\text { c. } \\
{\left[496 \_497 \text { delCT]+ }\right.} \\
\text { [430_431insGA] }\end{array}$ & $\begin{array}{l}\text { p. } \\
\text { [R166Afs]+ } \\
\text { [L144X] }\end{array}$ & $\mathrm{He}$ & $\begin{array}{l}\text { Xiang et } \\
\text { al.,(2018) }\end{array}$ \\
\hline 3 & 27 & Yemen & Yes & $46, X X$ & c. $[489$ C > G] & p. & ho & $\begin{array}{l}\text { Al-Agha } \\
\text { et al., } \\
(2018)\end{array}$ \\
\hline 4 & 29 & Chinese & No & $46, X X$ & $\begin{array}{l}\text { c. }[597+1 G>T]+ \\
{[268 G>C]}\end{array}$ & $\begin{array}{l}\text { P.[splicing]+ } \\
\text { [p.D90H] }\end{array}$ & $\mathrm{He}$ & $\begin{array}{l}\text { This } \\
\text { study }\end{array}$ \\
\hline
\end{tabular}

\section{Conclusion}

In conclusion, we identified two novel variants in the PSMC3IP gene in a Chinese female with POI. This is the first time that PSMC3IP mutation causing POI has been reported in Chinese population. Our findings expands the genotypic spectrum of the PSMC3IP gene in POI patients and supports the application of NGS in the genetic diagnosis of POI. Moreover, this finding sheds new light on genetic counseling, diagnosis, and therapy in POI.

\section{Declarations}




\section{Acknowledgments}

We thank the participating family members for their assistance and support.

\section{Authors' contributions}

Libin Mei and Lingling Huang carried out research design, experiments, analyzed data, explained the results, and drafted manuscripts. Yanru Huang participated in the design and interpretation of the research results. XueMei He was responsible for ultrasound examination and interpretation of the results. Xiaoling Wu and Huang He were involved in the experiment. Zhiying Su and Ping Li participated in the supervision, revising manuscript andits critical review.

\section{Funding}

This study was supported by the Fujian Medical Innovation Project(No.2018-CXB-29), the National Natural Science Foundation of China (Grant No. 31801044) and the Young and Middle-aged Personnel Training Program of Fujian Province University (Grant No. 2018-ZQN-90).

\section{Availability of data and materials}

The datasets used and/or analyzed during the current study are available from the corresponding author on reasonable request.

\section{Ethics approval and consent to participate}

This study fully complied with the tenets of the Declaration of Helsinki and has been approved by the Ethics Board of the Women's and children's Hospital affiliated to Xiamen University, China. Informed consent was obtained from all participants before testing.

\section{Consent for publication}

Not applicable.

\section{Competing interests}

The authors declare that they have no conflict of interest.

\section{Author details}

1Women and Children's Hospital, School of Medicine, Xiamen university.Xiamen, Fujian361003, China. 2Xiamen Key Laboratory of Reproduction and Genetics, Xiamen, Fujian361003, China. 3School of Public Health, Xiamen Universit, Xiamen, Fujian361102, China.

\section{Reference}

1. Nelson LM. Clinical practice. Primary ovarian insufficiency. N Engl J Med. 2009;360(6):606-14.

2. Tucker EJ , Grover SR, Bachelot A, Touraine P, Sinclair AH.Premature ovarian insufficiency: new perspectives on genetic cause and phenotypic spectrum. Endocr Rev. 2016;37(6):609-35. 
3. Gowri V, Al Shukri M, Al-Farsi FA, Al-Busaidi NA, Dennison D, Al Kindi S, et al. Aetiological profile of women presenting with premature ovarian failure to a single tertiary care center in Oman. Post Reprod Health. 2015;21(2):63-68.

4. Webber L, Davies M, Anderson R, Bartlett J, Braat D, Cartwright B, et al. ESHRE Guideline: Management of Women With Premature Ovarian Insufficiency.Hum Reprod. 2016;31(5):926-37.

5. Jiao X, Ke H, Qin Y, Chen ZJ. Molecular Genetics of Premature Ovarian Insufficiency.Trends Endocrinol Metab. 2018;29(11):795-807.

6. Rossetti R, Ferrari I, Bonomi M, Persani L. Genetics of Primary Ovarian Insufficiency.Clin Genet. 2017;91(2):183198.

7. Huhtaniemi I, Hovatta O, La Marca A, Livera G, Monniaux D, Persani L, et al. Advances in the Molecular Pathophysiology, Genetics, and Treatment of Primary Ovarian Insufficiency.Trends Endocrinol Metab. 2018;29(6):400-419.

8. Zangen D, Kaufman Y, Zeligson S, Perlberg S, Fridman H, Kanaan M, et al. XX Ovarian Dysgenesis Is Caused by a PSMC3IP/HOP2 Mutation That Abolishes Coactivation of Estrogen-Driven Transcription.Am J Hum Genet. 2011;89(4):572-9.

9. Al-Agha AE, Ahmed IA, Nuebel E, Moriwaki M, Moore B, Peacock KA, et al. Primary Ovarian Insufficiency and Azoospermia in Carriers of a Homozygous PSMC3IP Stop Gain Mutation.J Clin Endocrinol Metab. 2018;103(2):555-563.

10. Yang X, Touraine P, Desai S, Humphreys G, Jiang H, Yatsenko A,et al. Gene Variants Identified by Whole-Exome Sequencing in 33 French Women With Premature Ovarian Insufficiency.J Assist Reprod Genet. 2019;36(1):39-45.

11. Enomoto R, Kinebuchi T, Sato M, Yagi H, Kurumizaka H, Yokoyama S. Stimulation of DNA Strand Exchange by the Human TBPIP/Hop2-Mnd1 Complex.J Biol Chem. 2006;281(9):5575-81.

12. Schubert S, Ripperger T, Rood M, Petkidis A, Hofmann W, Frye-Boukhriss H, et al. GT198 (PSMC3IP) Germline Variants in Early-Onset Breast Cancer Patients From Hereditary Breast and Ovarian Cancer Families.Genes Cancer. 2017;8(1-2):472-483.

13. Achyut BR, Zhang H, Angara K, Mivechi NF, Arbab AS, Ko L. Oncoprotein GT198 Vaccination Delays Tumor Growth in MMTV-PyMT Mice.Cancer Lett. 2020 28;476:57-66.

14. Capdevila-Busquets E, Badiola N, Arroyo R, Alcalde V, Soler-López M, Aloy P. Breast Cancer Genes PSMC3IP and EPSTI1 Play a Role in Apoptosis Regulation.PLoS One. 2015 Jan 15;10(1):e0115352.

15. Tanaka T, Nakamura T, Takagi H, Sato M. Molecular Cloning and Characterization of a Novel TBP-1 Interacting Protein (TBPIP):enhancement of TBP-1 Action on Tat by TBPIP.Biochem Biophys Res Commun. 1997;239(1):17681.

16. Ko L, Cardona GR, Henrion-Caude A, Chin WW. Identification and Characterization of a Tissue-Specific Coactivator, GT198, That Interacts With the DNA-binding Domains of Nuclear Receptors.Mol Cell Biol. 2002;22(1):357-69.

17. Petukhova GV, Romanienko PJ, Camerini-Otero RD. The Hop2 Protein Has a Direct Role in Promoting Interhomolog Interactions During Mouse Meiosis.Dev Cell. 2003;5(6):927-36.

18. Leu JY, Chua PR, Roeder GS. The Meiosis-Specific Hop2 Protein of S. Cerevisiae Ensures Synapsis Between Homologous Chromosomes.Cell. 1998;94(3):375-86.

\section{Figures}




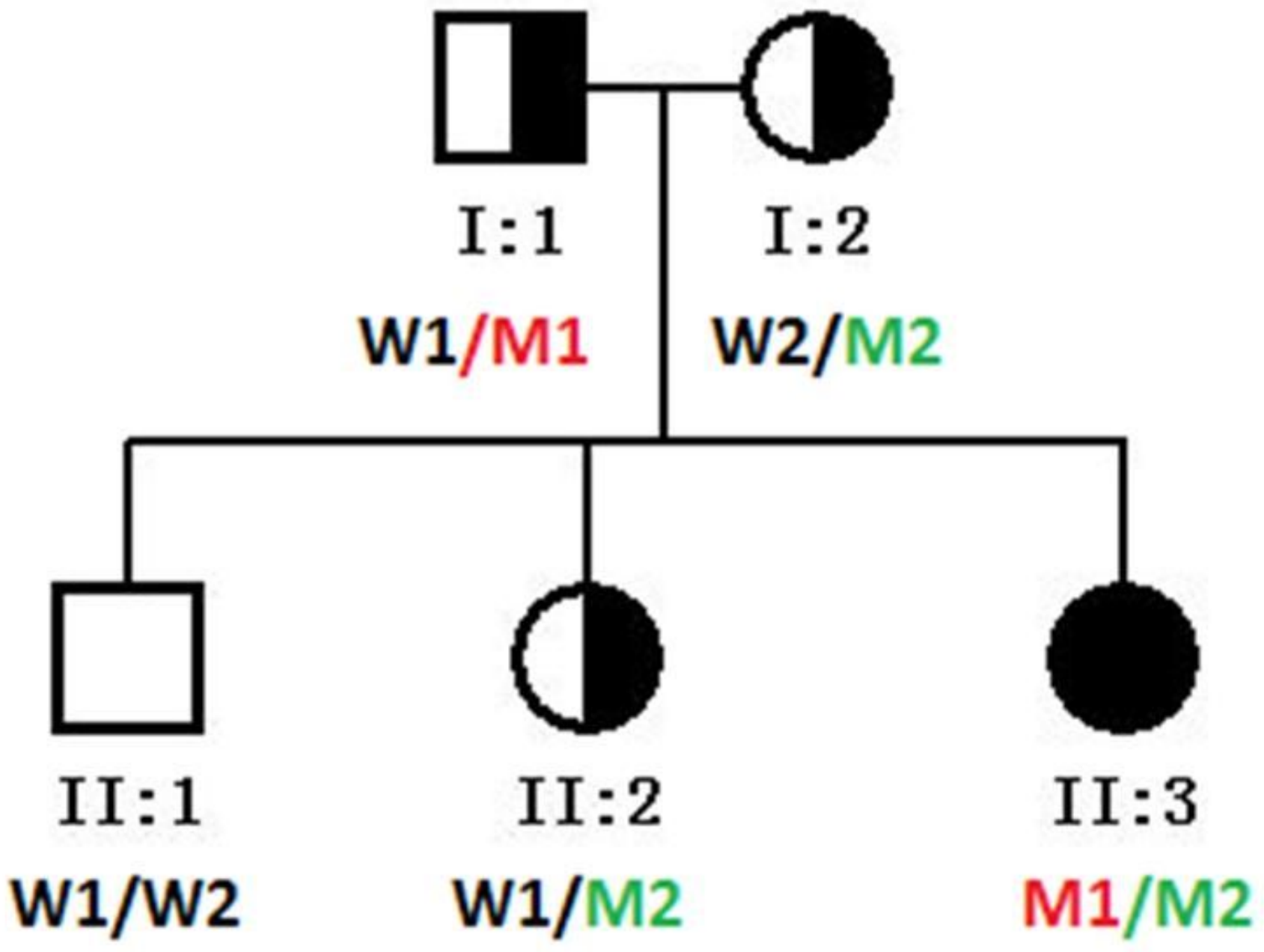

Figure 1

A. Pedigree of the family with POI. W, wild type; M1, c.597+1G>T mutation; M2, c. $268 \mathrm{G}>\mathrm{C}$ mutation; the proband is identified by an arrow 


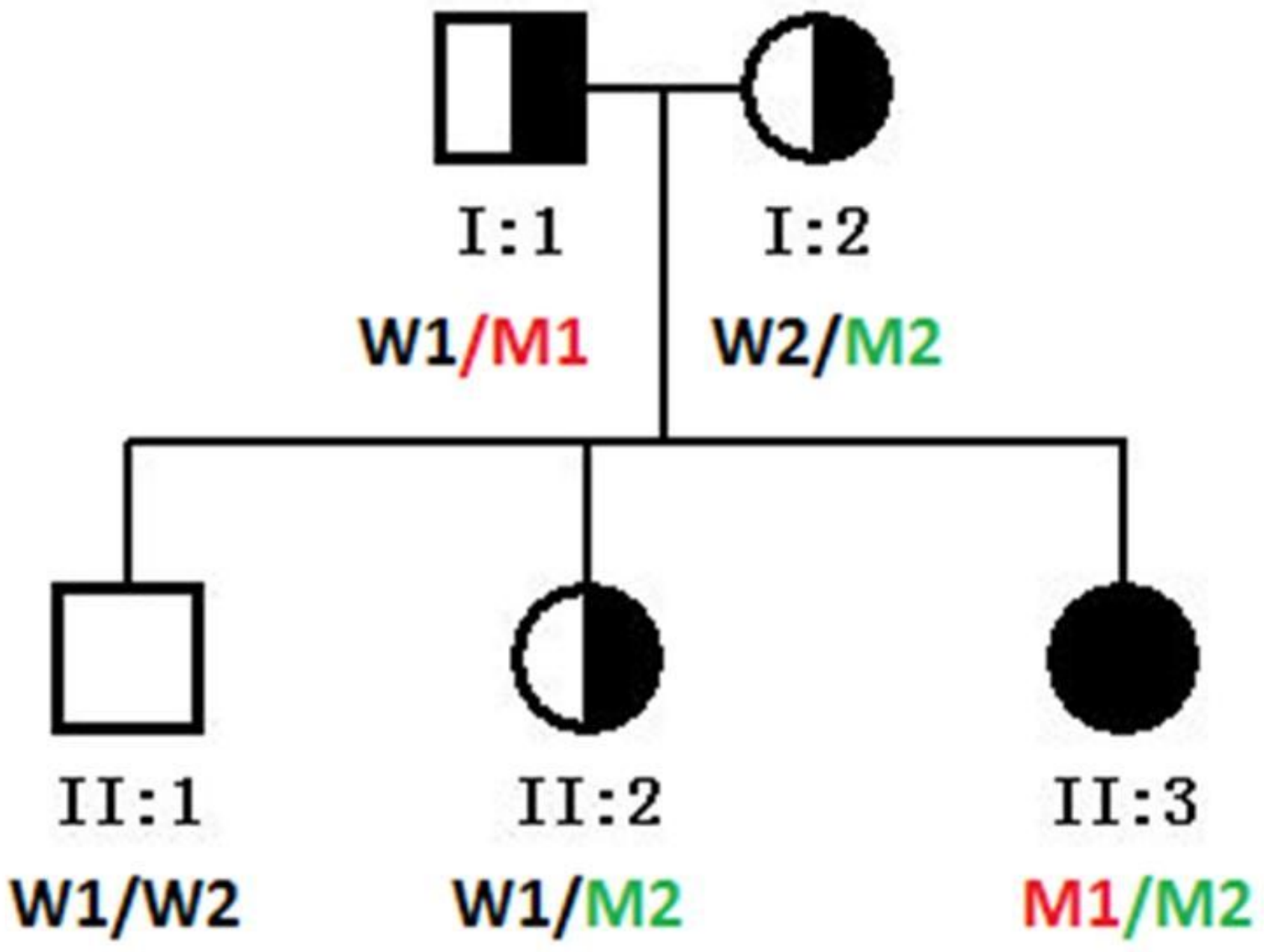

Figure 1

A. Pedigree of the family with POI. W, wild type; M1, c.597+1G>T mutation; M2, c. $268 \mathrm{G}>\mathrm{C}$ mutation; the proband is identified by an arrow 


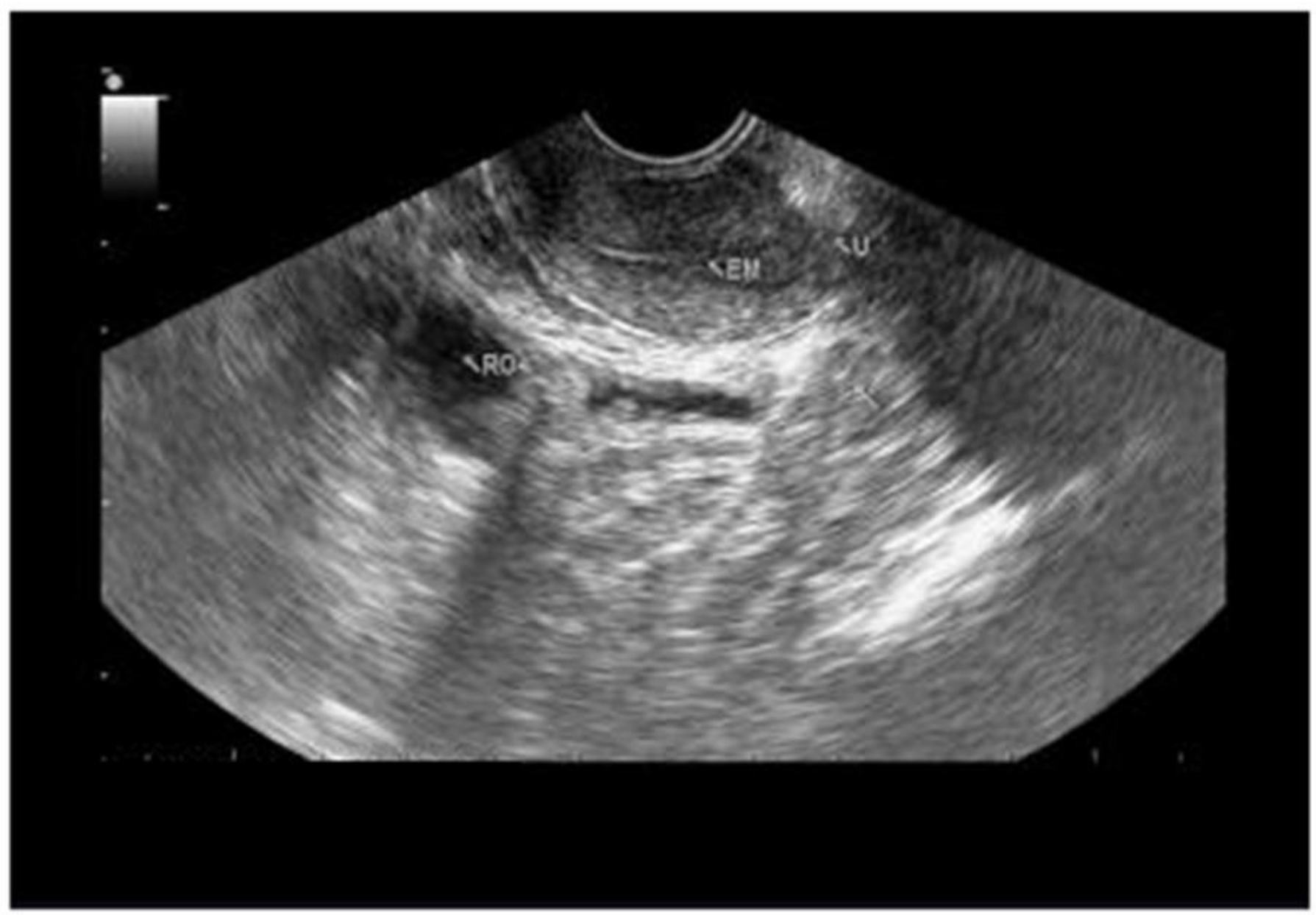

\section{Figure 2}

Transvaginal ultrasound examination showed that the uterus was small, and there were clues like changes in bilateral ovaries 


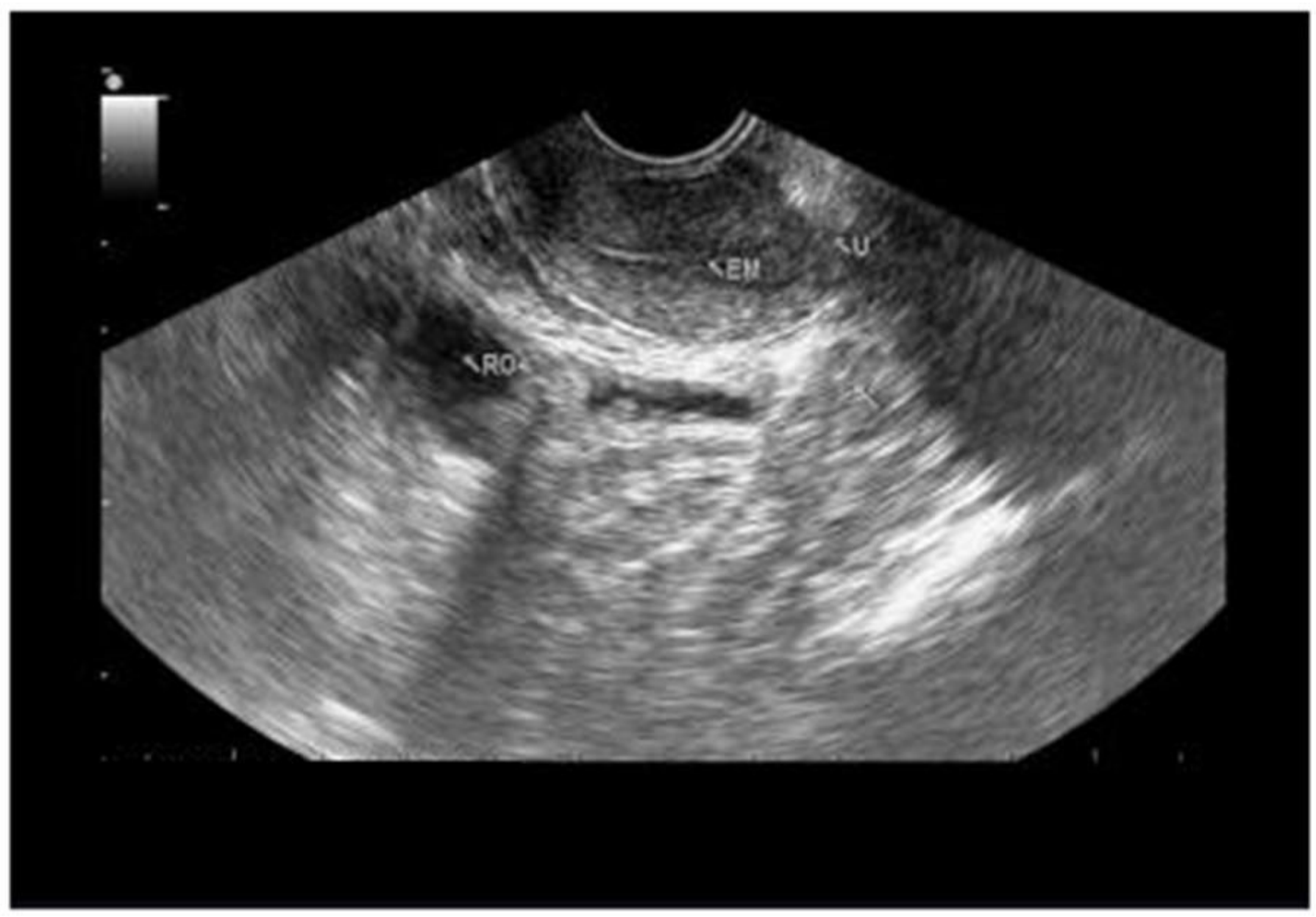

\section{Figure 2}

Transvaginal ultrasound examination showed that the uterus was small, and there were clues like changes in bilateral ovaries 

c. $597+1 G>T$
c. $268 \mathrm{G}>\mathrm{C}$
A G I I C I I I G I A A G I G
A G I C C C I A If A I G G C $\stackrel{150}{14}$ A

Patient
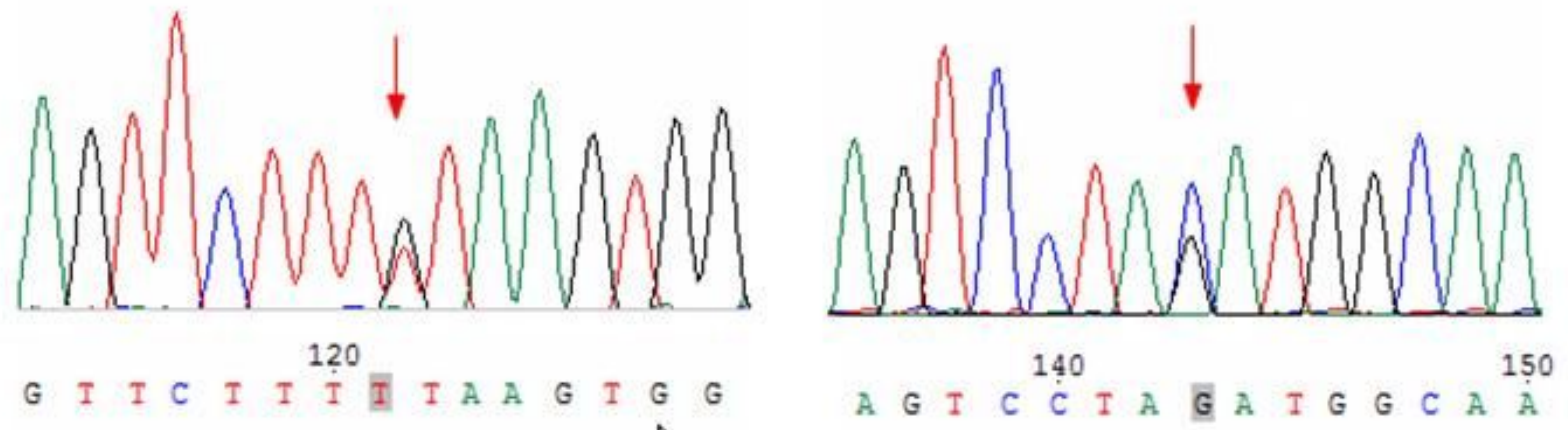

Father

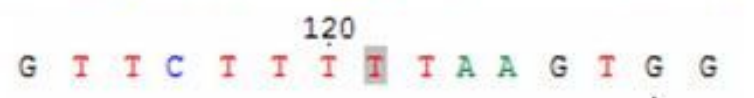

\section{Mother}
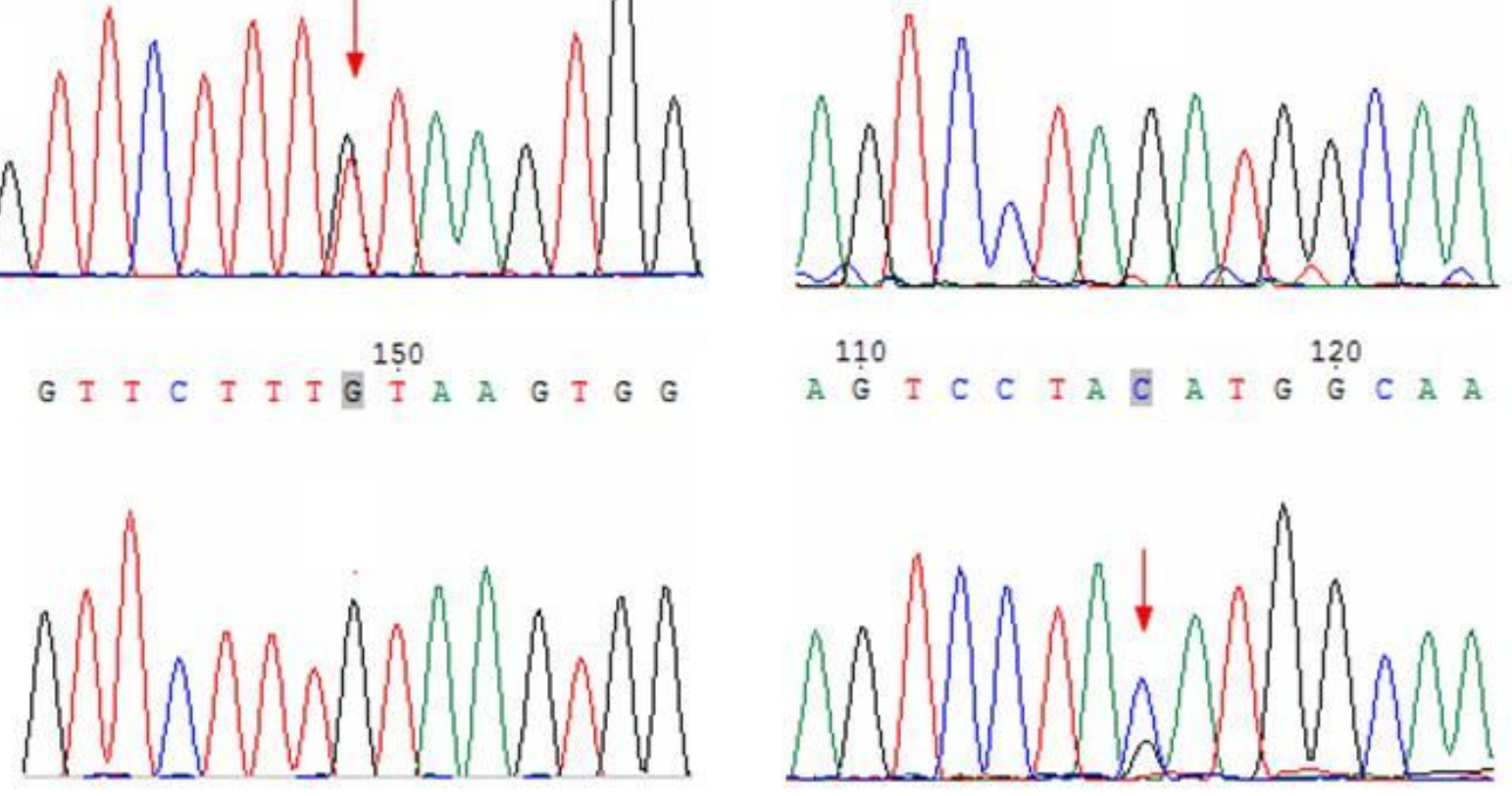

Figure 3

Identifying mutations in PSMC3IP. Electropherogram analysis of PSMC3IP in the proband showing compound heterozygous c.597+1G>T and c.268G >C mutations of PSMC3IP. The father (11) carried c.597+1G>T, while the mother (12) carried c. $268 \mathrm{G}>\mathrm{C}$ 

c. $597+1 G>T$
c. $268 \mathrm{G}>\mathrm{C}$
A $G$ I I C I I I G I A A G I G G A G I C C I A N A I G G

Patient
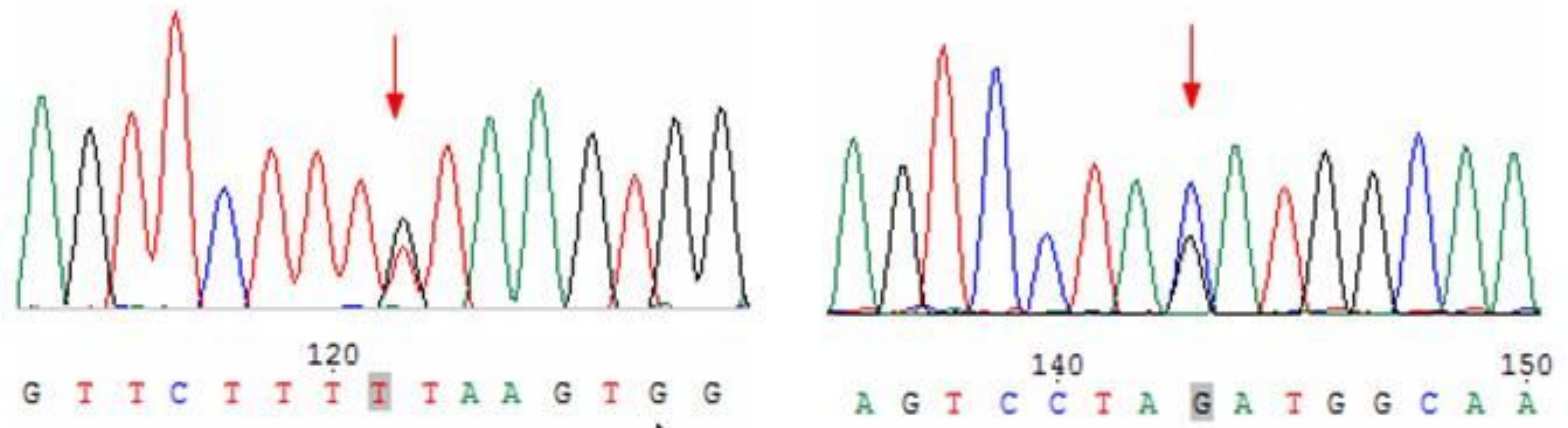

Father
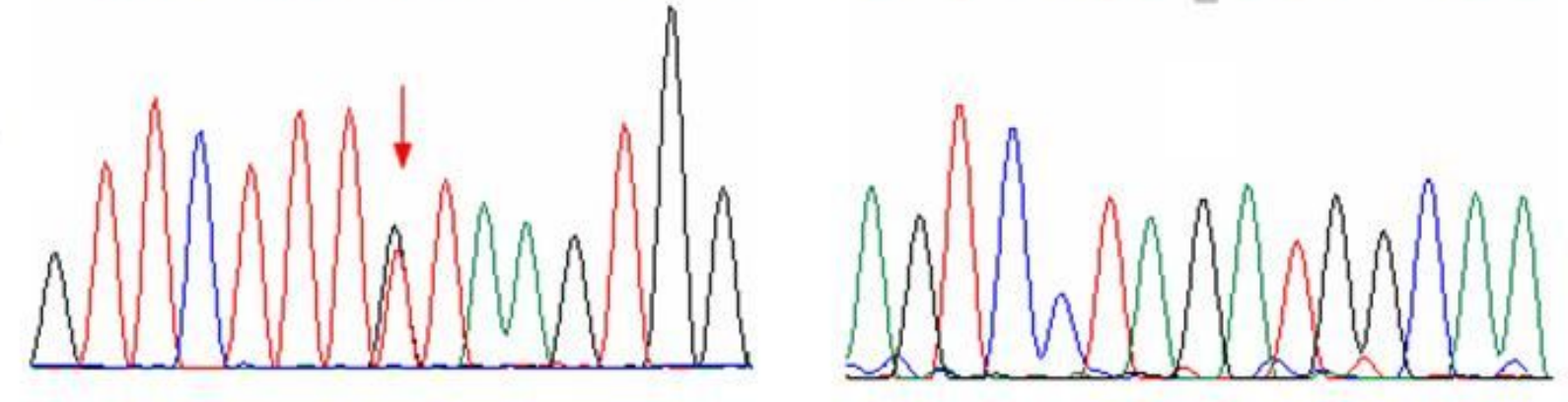

$$
\text { G I I C I I I G I I A A G I G G }
$$

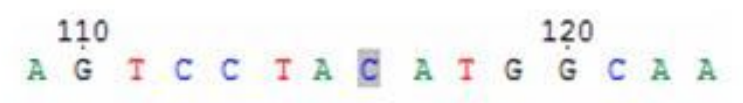

Mother
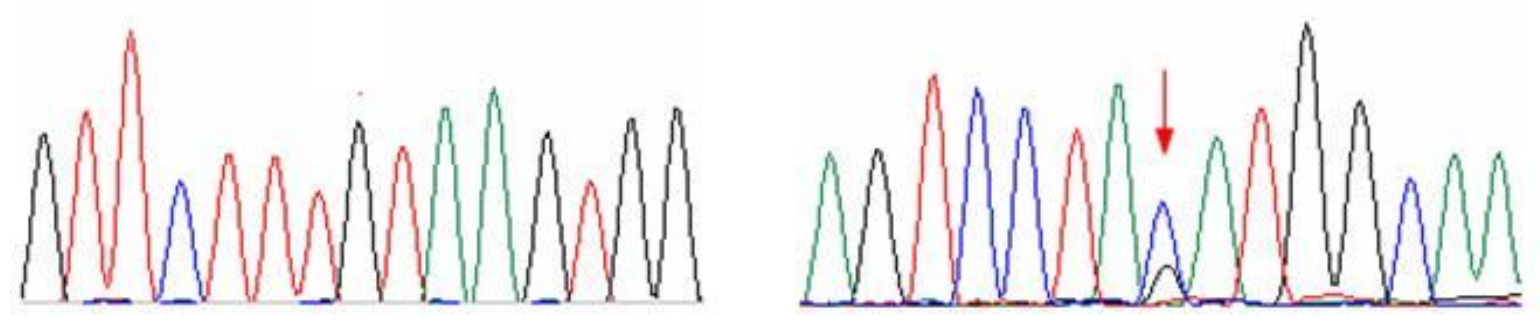

\section{Figure 3}

Identifying mutations in PSMC3IP. Electropherogram analysis of PSMC3IP in the proband showing compound heterozygous c.597+1G>T and c. $268 \mathrm{G}>\mathrm{C}$ mutations of PSMC3IP. The father (11) carried c.597+1G $>\mathrm{T}$, while the mother (12) carried c. $268 \mathrm{G}>\mathrm{C}$ 
This mutation is predicted to be PROBABLY DAMAGING with a score of 1.000 (sensitivity: 0.00 ; specificity: 1.00 )
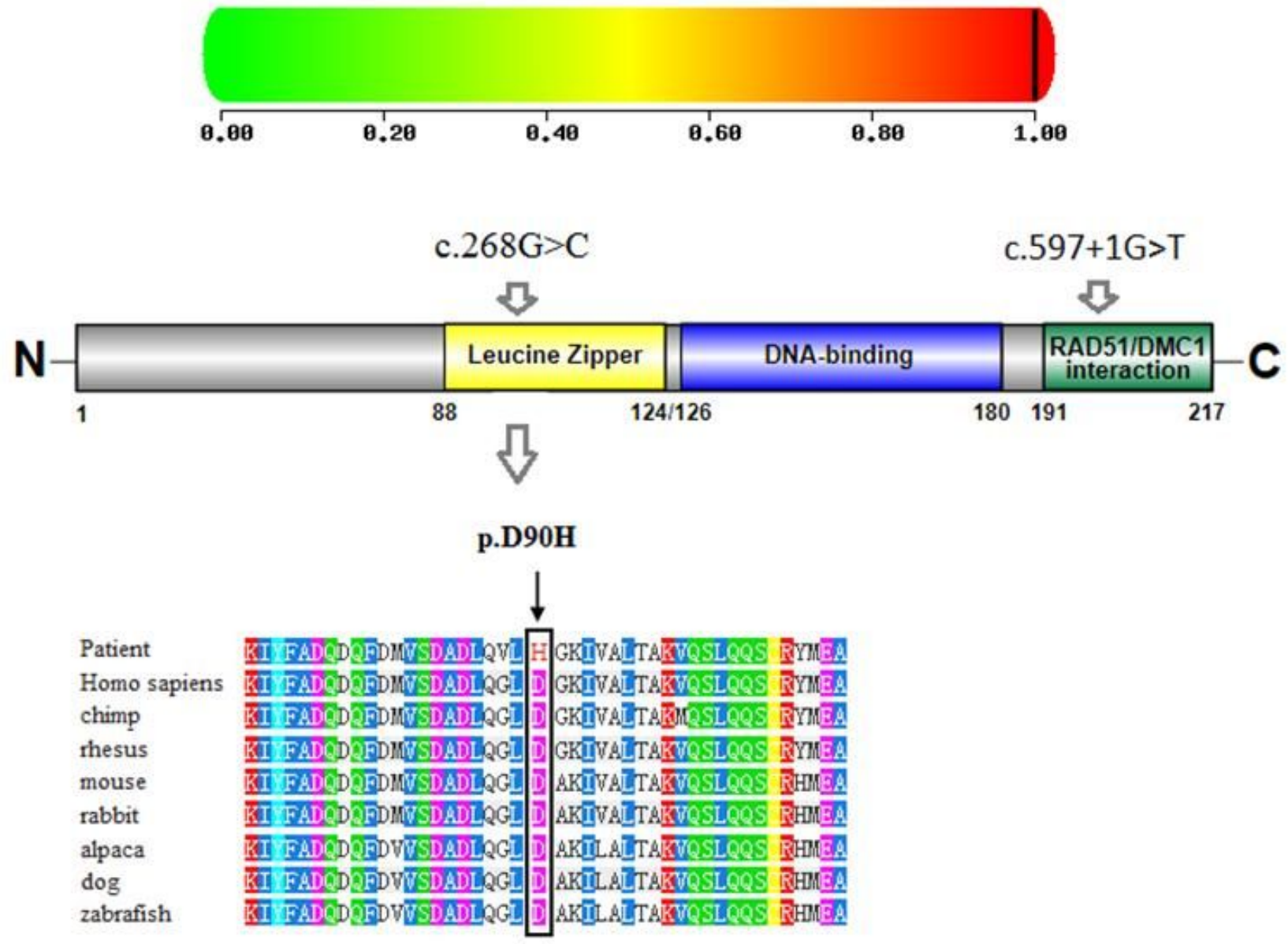

Figure 4

a) PolyPhen-2 reports for the pathogenicity of the amino acid substitution p.D90H in PSMC3IP. b) Diagram of PSMC3IP with predicted locations of protein sequence changes. c) Protein alignment showed that PSMC3IP p.D90H residues were conserved across multiple species, thus the two mutations occurred at an evolutionarily conserved amino acid 
This mutation is predicted to be PROBABLY DAMAGING with a score of 1.000 (sensitivity: 0.00 ; specificity: 1.00 )
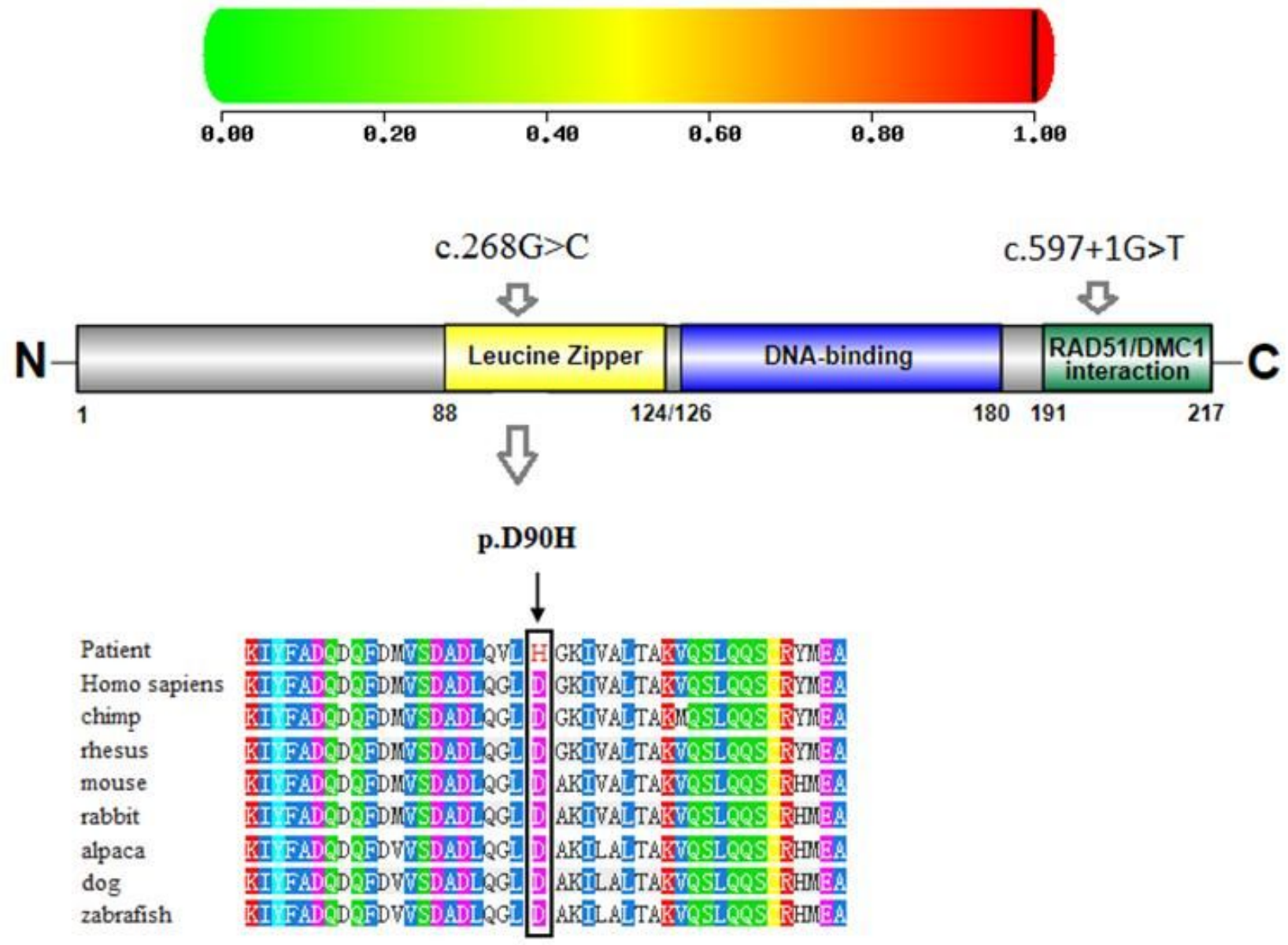

Figure 4

a) PolyPhen-2 reports for the pathogenicity of the amino acid substitution p.D90H in PSMC3IP. b) Diagram of PSMC3IP with predicted locations of protein sequence changes. c) Protein alignment showed that PSMC3IP p.D90H residues were conserved across multiple species, thus the two mutations occurred at an evolutionarily conserved amino acid

\section{Supplementary Files}

This is a list of supplementary files associated with this preprint. Click to download.

- SupplementaryTable1.xlsx

- SupplementaryTable1.xlsx 